\title{
Spotlight on Pimavanserin Tartrate and Its Therapeutic Potential in the Treatment of Major Depressive Disorder: The Evidence to Date
}

This article was published in the following Dove Press journal:

Drug Design, Development and Therapy

\author{
Vicki Soogrim ${ }^{1, *}$ \\ Valerie L Ruberto (D) ${ }^{\text {,** }}$ \\ James Murrough ${ }^{1,2}$ \\ Manish Kumar Jha (iD) 1,2 \\ 'Depression and Anxiety Center for \\ Discovery and Treatment, Department of \\ Psychiatry, Icahn School of Medicine at \\ Mount Sinai, New York, NY, USA; \\ ${ }^{2}$ Department of Neuroscience, Icahn \\ School of Medicine at Mount Sinai, \\ New York, NY, USA
}

*These authors contributed equally to this work
Correspondence: Manish Kumar Jha Depression and Anxiety Center for Discovery and Treatment, Department of Psychiatry, Icahn School of Medicine at Mount Sinai, I Gustave L. Levy Place,

New York, NY 10029, USA

Tel + I 2I2-585-4636

Email manish.jha@mssm.edu

\begin{abstract}
Major depressive disorder (MDD) is widely prevalent and one of the leading causes of disability. Treatment outcomes remain suboptimal with 1 in 3 patients with MDD responding inadequately to commonly used antidepressants. Pimavanserin, an atypical antipsychotic that modulates serotonergic neurotransmission by selectively binding to serotonin receptor (2A and $2 \mathrm{C}$ ) subtypes and without dopaminergic activity, may have the potential as an adjunctive treatment for MDD. In a phase 2 trial $(n=203)$, addition of pimavanserin, as compared to placebo, to stable treatment with antidepressants was associated with greater reduction in 17-item Hamilton Depression Rating Scale score [HAMD, least square means (95\% confidence interval) of $-1.7(-0.03,-3.37), \mathrm{p}=0.039]$. Furthermore, treatment with pimavanserin was associated with significantly greater improvement in specific symptoms associated with depression such as impaired sexual function, anxiety, sleepiness, and irritability. However, the availability of pimavanserin for clinical care of patients with MDD remains uncertain. Top-line results of phase 3 studies $(n=298)$ that were announced by the sponsor found similar reductions in HAMD (mean baseline-to-week-5 reduction of 9.0 and $8.1, \mathrm{p}=0.296)$ and rates of adverse events $(58.1 \%$ and $54.7 \%)$ with addition of pimavanserin and placebo respectively to stable treatment with antidepressants. Given the potential benefit for specific symptoms such as impaired sexual function, anxiety and sleep/wakefulness disturbances, future studies that enrich for these symptoms may be needed to clarify the utility of adjunctive pimavanserin in treatment of patients with MDD.
\end{abstract}

Keywords: major depressive disorder, pimavanserin, depression, antidepressant

\section{Introduction}

Major depressive disorder (MDD) affects 1 in 5 adults during their lifetime and is the second leading cause of disability in the United States. ${ }^{1,2}$ It is associated with impairments in multiple domains of life such as work productivity, social functions, day to day activities, and quality of life. ${ }^{3-6}$ Commonly used antidepressants are often ineffective in patients with $\mathrm{MDD} ;^{7}$ thereby necessitating the use of augmentation/adjunctive agents such as atypical antipsychotics ${ }^{8}$ and esketamine, ${ }^{9}$ psychotherapy, and/or non-invasive brain stimulation. ${ }^{10-13}$ Pimavanserin tartrate, hereafter referred to as pimavanserin, is one such drug that has been studied for improving depression and associated symptoms in patients with MDD who have responded inadequately to one or more courses of serotonergic antidepressant. ${ }^{14-16}$ Approved by the Food and Drug Administration (FDA) for treatment of hallucinations and delusions associated with Parkinson's disease psychosis, ${ }^{17}$ pimavanserin 
differs from other atypical antipsychotics in having no appreciable affinity for dopaminergic receptors and from selective serotonin reuptake inhibitors in having no appreciable affinity for serotonin transporter. ${ }^{18}$ In this regard, it represents a new class of medications that selectively targets specific serotonin receptor subtype(s). ${ }^{19}$

We have focused this review on the therapeutic potential of pimavanserin for MDD. We have first provided a brief overview of clinical pharmacology of pimavanserin and its use in Parkinson's disease psychosis. We then review the findings of a recently-completed phase 2 double-blind randomized placebo-controlled trial of pimavanserin and specifically discuss its utility in improving symptom domains such as sexual dysfunction, anxiety and irritability that are typically unaffected or even worsened by commonly used serotonergic antidepressants. ${ }^{14,15,20}$ Finally, we discuss the top-line results in media from the sponsor for the phase 3 studies of adjunctive pimavanserin for patients with MDD.

\section{Clinical Pharmacology of Pimavanserin}

Pimavanserin, $N$-(4-Fluorophenylmethyl)- $N$-(1-methylpi peridin-4-yl)- $N^{\prime}-(4-(2-m e t h y l p r o p y l-o x y)$ phenylmethyl) Carbamide $(2 R, 3 R)$-Dihydroxybutanedioate $(2: 1),^{21}$ is a small molecule (freebase molecular weight of 427.6 $\mathrm{g} / \mathrm{mol})^{17}$ that specifically modulates serotoninergic neurotransmission as an inverse agonist/antagonist of the 5-hydroxytryptamine $2 \mathrm{~A}\left(5-\mathrm{HT}_{2 \mathrm{~A}}\right)$ and $5-\mathrm{HT}_{2 \mathrm{C}}$ (at higher doses) receptors. ${ }^{21}$ In radioligand binding studies, pimavanserin shows no appreciable binding for any other serotoninergic, dopaminergic, adrenergic, histamine, or muscarinic receptors or to serotonin or norepinephrine transporters. $^{21}$ Therefore, its pharmacological effect is driven predominantly by its effect on $5-\mathrm{HT}_{2 \mathrm{~A}}$ receptors and to a lesser extent on $5-\mathrm{HT}_{2 \mathrm{C}}$ receptors.

Recommended daily dose of pimavanserin for Parkinson's disease psychosis is $34 \mathrm{mg}$ freebase that is contained in $40 \mathrm{mg}$ of tartrate salt. Pimavanserin demonstrates dose-proportional pharmacokinetics after single oral doses from 17 to $255 \mathrm{mg}$ (0.5- to 7.5-times the recommended dosage). Mean plasma half-lives of pimavanserin and it's major circulating active metabolite ( $N$-desmethylated metabolite) are 57 hours and 200 hours, respectively. ${ }^{17}$ Pimavanserin is predominantly metabolized by CYP3A4 and CYP3A5 and is not known to be affected by other cytochrome P450 enzymes. Therefore, drugs such as itraconazole, ketoconazole, clarithromycin, and indinavir increase the levels of pimavanserin by inhibiting CYP3A4 and may warrant dose reduction whereas CYP3A4 inducers such as carbamazepine, St. John's wort, phenytoin, and rifampin decreased the levels of pimavanserin and should be avoided. ${ }^{17}$ Levels of pimavanserin are not affected by food.

\section{Use of Pimavanserin for Parkinson's Disease Psychosis}

In April 2016, pimavanserin became the first FDA-approved treatment for Parkinson's disease psychosis and provided alternatives to commonly used antipsychotics that either worsened motor symptoms or were ineffective. ${ }^{22-24}$ While low dose clozapine was shown to improve psychosis symptoms without worsening motor symptoms, it's use is limited by adverse side effects including sedation, hyper salivation, hypotension, and a small but potentially fatal risk of agranulocytosis. ${ }^{23}$ Quetiapine, another atypical antipsychotic, did not show to be more efficacious than placebo in four randomized controlled clinical trials. ${ }^{25}$

Therapeutic effects of pimavanserin were first evaluated in a phase 2 4-week dose-escalation clinical trial of 60 patients with Parkinson's disease psychosis, 29 of whom received pimavanserin while 31 received placebo. Patients in the pimavanserin arm had the option of increasing the study drug to $40 \mathrm{mg}$ on day 8 and $60 \mathrm{mg}$ on day 15 . The study found that pimavanserin did not differ from placebo in worsening of motor function, sedation or hypotension. ${ }^{26}$ Furthermore, those treated with pimavanserin showed significantly greater improvement in some but not all measures of psychosis including in measures of hallucinations and delusions. $^{26} \mathrm{~A}$ recent meta-analysis of four pimavanserin trials (total $n=638$ ) found significantly greater improvement in psychosis symptoms with pimavanserin versus placebo. ${ }^{25}$ In these studies, common adverse effects with pimavanserin were peripheral edema $(7 \%)$ and confusional state $(6 \%){ }^{17}$ Clinical trials also found that pimavanserin prolongs the QT interval which may warrant greater caution and further evaluations (such as electrocardiogram) when added on to stable treatment with commonly used antidepressants that can prolong QT interval such as citalopram. ${ }^{17,27}$

\section{Pimavanserin as Adjunctive Therapy for MDD}

Given the potential synergistic effect of $5-\mathrm{HT}_{2 \mathrm{~A}}$ antagonism and activation of other 5 -HT receptors, ${ }^{28}$ antidepressant effects of pimavanserin were tested in a phase 2 study 
(CLARITY, NCT03018340). ${ }^{14}$ As reported by Fava and colleagues, this multicenter, randomized, double-blind, placebo-controlled study randomized 207 patients with MDD at 27 sites between December 2016 to October 2018. ${ }^{14}$ Eligible patients were males and females aged $\geq 18$ years with a body mass index between 18 to 35 and were in a current depressive episode of moderate severity at both screening and baseline visits. ${ }^{14}$ Furthermore, eligibility was restricted to patients $(n=207)$ who had an inadequate response to 1 or 2 antidepressant treatments during the current depression episode and were receiving treatment with exactly 1 of the following drugs at approved doses for at least 8 weeks (stable dose for 4 weeks): citalopram, desvenlafaxine, duloxetine, escitalopram, fluoxetine, paroxetine, sertraline, venlafaxine, or venlafaxine extended-release. ${ }^{14}$ The study utilized a 2-stage Sequential Parallel-Comparison Design ${ }^{29}$ whereby patients were randomized to a 10 -week doubleblind treatment period. During Stage 1, patients were randomized to placebo or pimavanserin in a 3:1 ratio for 5 weeks. At the end of 5 weeks, non-responders to placebo were re-randomized to placebo or pimavanserin in a $1: 1$ fashion for an additional 5 weeks (Stage 2). All patients randomized to pimavanserin in Stage 1 continued it in Stage 2, whereas responders to placebo in Stage 1 remained on placebo in Stage 2. ${ }^{14}$ During both stages, visits were conducted at weekly intervals. Patients continued their background antidepressant at a stable dose for the duration of the study. Patients and investigators were blinded to treatment assignment.

Using a weighted repeated measures model, ${ }^{30}$ analyses of data from Stages 1 and 2 showed greater improvement in overall depression severity [17-item Hamilton Depression Rating Scale (HAMD)]; least squares (LS) mean difference $(95 \%$ confidence interval, CI) $=-1.7(-0.03,-3.37), p=0.039]$ with addition of pimavanserin, as compared to placebo, to stable treatment with antidepressants. Furthermore, substantially more patients (all $\mathrm{p}<0.05$ ) randomized to pimavanserin attained response ( $\geq 50 \%$ reduction in HAMD from baseline) or remission (HAMD $\leq 7)$ than placebo from weeks 2 to 5 of Stage 1. During Stage 1, patients in pimavanserin arm had greater improvement in psychosocial function (as assessed with Sheehan Disability Scale; Cohen's $\mathrm{d}$ effect size $=0.50, \mathrm{p}=0.0036)$ and in Clinical Global Impressions severity scale (Cohen's d effect size $=0.67$, $\mathrm{p}=0.0001) .{ }^{14}$
Adverse events related to treatment were reported by $48.1 \%(25 / 52)$ of patients in pimavanserin and $27.1 \%$ $(42 / 155)$ of patients in placebo group during Stage 1. The most common adverse events with pimavanserin were dry mouth, nausea, and headache. ${ }^{14}$ Use of pimavanserin was associated with low rates of extrapyramidal symptoms. The proportion of patients who discontinued treatment were similar in placebo $(1.9 \%$; $3 / 155)$ and pimavanserin $(1.9 \% ; 1 / 52)$ arms during Stage 1. There was greater reduction in suicidal ideation (assessed with single item of HAMD) with pimavanserin vs placebo at week 3 (Cohen's d effect size: $0.431, p=0.012$ ). Suicidal ideation at post-baseline visits as measured with Columbia Suicide Severity Rating Scale $^{31}$ were reported by $18.1 \%$ (28/155) and $17.3 \%$ (9/52) of patients in placebo and pimavanserin arms respectively during Stage 1 and $20.7 \%$ (6/29) and $13.8 \%(4 / 29)$ of patients in placebo and pimavanserin arms respectively during Stage 2. ${ }^{32}$ During Stage 2, $20.7 \%$ (6/29) patients in pimavanserin arms did not experience any clinically relevant changes in vital signs or electrocardiogram. While pre-to-post-treatment changes in plasma glucose were similar in pimavanserin and placebo arms, there was significantly greater reduction in prolactin with pimavanserin versus placebo [-28.4 (104.7) vs $10.2(106.7) \mu \mathrm{IU} / \mathrm{mL}] .{ }^{14}$

\section{Effect of Pimavanserin on Symptoms Associated with Depression Impaired Sexual Function}

Impaired sexual functioning is common in patients with MDD and may occur as a side-effect of commonly used antidepressants. $^{33,34}$ Impaired sexual function is associated with poorer quality of life, especially in patients with MDD who have experienced inadequate improvement with an initial course of serotonergic antidepressant. ${ }^{35}$ Therefore, Freeman and colleagues undertook a secondary analysis of CLARITY data to evaluate the effect of pimavanserin on sexual functioning. ${ }^{15}$ They found that use of pimavanserin $(n=51)$ from baseline-to-week-5 was associated with significantly greater improvement [LS mean $(95 \% \mathrm{CI})$ difference $=-0.63 \quad(-0.96,-0.30)$, Cohen's $\mathrm{d}=0.61$, $\mathrm{p}=0.0002$ )] in sexual functioning (quantified with the Massachusetts General Hospital Sexual Functioning Index $)$ versus placebo $(\mathrm{n}=152) .{ }^{15}$ Even when analyzed across the 10-week study duration, improvement in sexual 
functioning with pimavanserin was significantly greater than placebo [ $(\mathrm{LS}$ mean $(95 \% \mathrm{CI})=-0.47(-0.72,-0.22)$, $\mathrm{p}=0.0003)] .{ }^{15}$ Furthermore, the authors found significant improvement with pimavanserin vs placebo in the sexual functioning as assessed with the single item (14th) of HAMD. ${ }^{15}$

\section{Anxiety}

Symptoms of anxiety are common in patients with MDD and are associated with poorer prognosis including impaired functioning, reduced likelihood of clinically significant improvement, an increased incidence of side effects, and a slower rate of recovery from a depressive episode. ${ }^{36,37}$ Furthermore, use of antidepressants may worsen symptoms of anxiety which in turn is associated with lower likelihood of subsequent remission. ${ }^{20}$ A secondary analysis of the CLARITY study specifically looked at 1) the changes in symptoms of anxiety (as measured with the anxiety/somatization factor of HAMD) ${ }^{36}$ with pimavanserin versus placebo and 2) differences in remission (HAMD $\leq 7)$ and response ( $\geq 50 \%$ reduction in HAMD from baseline) rates between the two treatment arms based on presence of anxious features (HAMD anxiety/ somatization factor score $\geq 7$ ) at baseline. ${ }^{16}$ Papakostas and colleagues found significantly greater improvement in scores of HAMD anxiety/somatization factor with pimavanserin vs placebo [LS mean (standard error, SE) of difference $=-1.5(0.41)$, Cohen's d effect size $=0.63$, $\mathrm{p}=0.0003]$. Presence of anxious features at baseline also predicted significantly higher response (pimavanserin = $55.2 \%$, placebo $=22.4 \%, \mathrm{p}=0.001$, number needed to treat $=3.0$ ) and remission (pimavanserin $=24.1 \%$, placebo $=5.3 \%, \mathrm{p}=0.005$, number needed to treat $=5.3$ ) rates during Stage 1 of CLARITY. ${ }^{16}$

\section{Sleep/Wakefulness Disturbances}

Up to $90 \%$ of patients with MDD report sleep disturbances which in turn are associated with poorer treatment outcomes such as failure to attain remission and increased risk of relapse/recurrent after initial symptomatic improvement. ${ }^{38,39}$ Excessive sleepiness, a disabling feature of depression, ${ }^{40}$ was assessed with the Karolinska sleepiness scale $(\mathrm{KSS})^{41}$ at each visit of the CLARITY study. ${ }^{14}$ From baseline to week 5 of Stage 1 of CLARITY, there was significantly greater reduction (Cohen's d effect size $=0.63, p=0.0003)$ in KSS scores with pimavanserin [LS means $(\mathrm{SE})=-1.7(0.260]$ than with placebo [LS means $(\mathrm{SE})=-0.60(0.15)]^{42}$

\section{Irritability}

Elevated irritability after antidepressant initiation is associated with poorer longer-term treatment outcomes such as reduced likelihood of achieving remission and persistently elevated suicidal ideation. ${ }^{43-46}$ Secondary endpoints from the CLARITY study show that adjunctive use of pimavanserin is associated with greater reduction (Cohen's $d$ effect size $=0.56, p=0.001)$ in irritability than placebo [LS means $(\mathrm{SE})=-19.5(2.17)$ vs $-11.2(1.28)]$.

\section{Preliminary Findings from Phase 3 Studies}

Due to the promising results from phase 2 trial of pimavanserin, phase 3 trials were initiated in the United States and Europe (NCT03968159 and NCT03999918). In July 2020, the sponsor announced top-line results that combined data from these two trials (total $n=298$ ) and showed that the primary endpoint, which was HAMD score change from baseline to week 5, did not achieve statistical significance (mean baseline-to-week-5 reduction in HAMD score of 9.0 and 8.1 with pimavanserin and placebo respectively, $\mathrm{p}=0.296) .{ }^{47}$ Among secondary outcomes, greater improvement with pimavanserin was observed for Clinical Global Impressions severity scale $(\mathrm{p}=0.042)$ and subjective sleepiness as quantified by KSS $(p=0.005) .{ }^{47}$ Rates of adverse events were similar in pimavanserin (58.1\%) and placebo (54.7\%) arms according to the top-line results that were announced by the sponsor. Rates of discontinuations due to adverse events were identical in both arms (2.7\%). Common side effects reported specifically with pimavanserin were diarrhea, dry mouth and headache. ${ }^{47}$ However, use of pimavanserin vs placebo was not associated with any clinically significant differences in vital signs, metabolic parameters or extrapyramidal symptoms. ${ }^{47}$

\section{Conclusion}

Pimavanserin is an atypical antipsychotic that selectively targets serotonin receptor subtypes $\left(5-\mathrm{HT}_{2 \mathrm{~A}}\right.$ and $\left.5-\mathrm{HT}_{2 \mathrm{C}}\right)$ and is approved by the FDA for treatment of Parkinson's disease psychosis. A recently completed phase 2 study showed that adjunctive use of pimavanserin may improve depression and associated symptoms in patients with MDD who have inadequately improved with 1-2 adequate courses of antidepressant treatments. However, the top-line results announced by the sponsor show that primary endpoint of greater HAMD score change with pimavanserin versus placebo from baseline to week 5 of phase 3 studies was not 
attained. Therefore, future availability of pimavanserin for clinical care of patients with MDD remains uncertain. Future prospective studies are needed to evaluate the role of pimavanserin in targeting specific symptom domains such as impaired sexual functioning, anxiety, and sleep disturbances that often occur in patients with MDD and may be worsened by antidepressant treatments and may consider utilizing strategies to reduce placebo response. ${ }^{29,48}$ In conclusion, pimavanserin is a novel atypical antipsychotic that may be useful as an adjunctive therapy in patients with MDD who have inadequately improved with their current antidepressant and may have specific symptoms associated with depression such as impaired sexual functioning, anxiety, and sleep disturbances.

\section{Acknowledgment}

Co-first authors: Vicki Soogrim and Valerie L Ruberto.

\section{Disclosure}

James Murrough reports personal fees from Allergan,personal fees from Boehringer Ingelheim, personal fees from Clexio Biosciences, personal fees from Fortress Biotech, personal fees from Global Medical Education (GME), personal fees from Otsuka, personal fees from Sage Therapeutics, and personal fees from Engrail Therapeutics, outside the submitted work. James Murrough is named on a patent pending for neuropeptide $\mathrm{Y}$ as a treatment for mood and anxiety disorders and on a patent pending for the use of KCNQ channel openers to treat depression and related conditions. Manish Kumar Jha reports grants from Acadia Pharmaceuticals, contracted research for Janssen Research \& Development, honoraria for continuing medical education preesntation from North American Center for Continuing Medical Education, and honoraria for continuing medical education presentation from Global Medical Education, during the conduct of the study; and their institution has received funds from Acadia Pharmaceuticals for the contract research that they conducted for evaluating safety and efficacy of pimavanserin in patients with major depressive disorder (MDD). Their institution, the Icahn School of Medicine at Mount Sinai is named on a patent and has entered into a licensing agreement and will receive payments related to the use of ketamine or esketamine for the treatment of depression. The Icahn School of Medicine at Mount Sinai is also named on a patent related to the use of ketamine for the treatment of PTSD. James Murrough and Manish Kumar Jha are not named on these patents and will not receive any payments. The authors report no other potential conflicts of interest for this work.

\section{References}

1. Hasin DS, Sarvet AL, Meyers JL, et al. Epidemiology of adult DSM-5 major depressive disorder and its specifiers in the United States. JAMA Psychiatry. 2018;75(4):336-346. doi:10.1001/ jamapsychiatry.2017.4602

2. Vos T, Abajobir AA, Abbafati C, Abbas KM, Abate K. Global, regional, and national incidence, prevalence, and years lived with disability for 328 diseases and injuries for 195 countries, 1990-2016: a systematic analysis for the global burden of disease study 2016 . Lancet. 2017;390(10100):1211-1259.

3. Jha MK, Greer TL, Grannemann BD, Carmody T, Rush AJ, Trivedi MH. Early normalization of quality of life predicts later remission in depression: findings from the CO-MED trial. $J$ Affect Disord. 2016;206:17-22. doi:10.1016/j.jad.2016.07.012

4. Jha MK, Minhajuddin A, Greer TL, Carmody T, Rush AJ, Trivedi MH. Early improvement in psychosocial function predicts longer-term symptomatic remission in depressed patients. PLoS One. 2016;11(12):e0167901. doi:10.1371/journal.pone.0167901

5. Jha MK, Minhajuddin A, Greer TL, Carmody T, Rush AJ, Trivedi MH. Early improvement in work productivity predicts future clinical course in depressed outpatients: findings from the CO-MED trial. Am J Psychiatry. 2016;173(12):1196-1204. doi:10.1176/appi.ajp.2016.16020176

6. Jha MK, Teer RB, Minhajuddin A, Greer TL, Rush AJ, Trivedi MH. Daily activity level improvement with antidepressant medications predicts long-term clinical outcomes in outpatients with major depressive disorder. Neuropsychiatr Dis Treat. 2017;13:803-813. doi:10.2147/NDT.S128407

7. Rush AJ, Trivedi MH, Wisniewski SR, et al. Acute and longer-term outcomes in depressed outpatients requiring one or several treatment steps: a STAR*D report. Am J Psychiatry. 2006;163(11):1905-1917. doi:10.1176/ajp.2006.163.11.1905

8. Ruberto VL, Jha MK, Murrough JW. Pharmacological treatments for patients with treatment-resistant depression. Pharmacy (Basel). 2020;13(6):6. doi:10.3390/ph13060116

9. Papakostas GI, Salloum NC, Hock RS, et al. Efficacy of esketamine augmentation in major depressive disorder: a meta-analysis. $J$ Clin Psychiatry. 2020;81(4):4. doi:10.4088/JCP.19r12889

10. Boes AD, Kelly MS, Trapp NT, Stern AP, Press DZ, Pascual-Leone A. Noninvasive brain stimulation: challenges and opportunities for a new clinical specialty. J Neuropsychiatry Clin Neurosci. 2018;30 (3):173-179. doi:10.1176/appi.neuropsych.17110262

11. Jha MK, Trivedi MH. Experimental therapies for treatment-resistant depression: "how do you decide when to go to an unproven or experimental therapy with patients that are treatment-resistant depression?". Focus (Am Psychiatr Publ). 2018;16(3):279-284. doi:10.1176/appi.focus.20180013

12. Malhi GS, Bell E, Singh A, et al. The 2020 royal Australian and New Zealand college of psychiatrists clinical practice guidelines for mood disorders: major depression summary. Bipolar Disord. 2020.

13. Park LT, Zarate CA Jr., Solomon CG. Depression in the primary care setting. $N$ Engl J Med. 2019;380(6):559-568. doi:10.1056/ NEJMcp1712493

14. Fava M, Dirks B, Freeman MP, et al. A phase 2, randomized, double-blind, placebo-controlled study of adjunctive pimavanserin in patients with major depressive disorder and an inadequate response to therapy (CLARITY). J Clin Psychiatry. 2019;80(6):6. doi:10.4088/ JCP. $19 \mathrm{~m} 12928$

15. Freeman MP, Fava M, Dirks B, et al. Improvement of sexual functioning during treatment of MDD with adjunctive pimavanserin: a secondary analysis. Depress Anxiety. 2020;37(5):485-495. doi:10.1002/da.23017 
16. Papakostas GI, Fava M, Freeman MP, et al. Effect of pimavanserin on anxious depression in patients with major depression and an inadequate response to previous therapy: secondary analysis of the clarity study. Int Clin Psychopharmacol. 2020;35(6):313-321. doi:10.1097/YIC.0000000000000328

17. Prescribing Information for Pimavanserin; 2019. Available from: https://www.nuplazid.com/sites/nuplazid/files/pdf/NUPLAZID_ Prescribing_Information.pdf. Accessed November 01, 2020.

18. Hacksell U, Burstein ES, McFarland K, Mills RG, Williams H. On the discovery and development of pimavanserin: a novel drug candidate for Parkinson's psychosis. Neurochem Res. 2014;39 (10):2008-2017. doi:10.1007/s11064-014-1293-3

19. Meltzer HY, Roth BL. Lorcaserin and pimavanserin: emerging selectivity of serotonin receptor subtype-targeted drugs. J Clin Invest. 2013;123(12):4986-4991.

20. Jha MK, Minhajuddin A, South C, Rush AJ, Trivedi MH. Worsening anxiety, irritability, insomnia, or panic predicts poorer antidepressant treatment outcomes: clinical utility and validation of the concise associated symptom tracking (CAST) scale. Int J Neuropsychopharmacol. 2018;21(4):325-332. doi:10.1093/ijnp/pyx097

21. Vanover KE, Weiner DM, Makhay M, et al. Pharmacological and behavioral profile of $\mathrm{N}$-(4-fluorophenylmethyl)-N-(1-methylpiperidin4-yl)-N'-(4-(2-methylpropyloxy)phenylmethyl) carbamide (2R,3R)dihydroxybutanedioate (2:1) (ACP-103), a novel 5-hydroxytryptamine (2A) receptor inverse agonist. J Pharmacol Exp Ther. 2006;317 (2):910-918. doi:10.1124/jpet.105.097006

22. Cummings J, Isaacson S, Mills R, et al. Pimavanserin for patients with Parkinson's disease psychosis: a randomised, placebo-controlled phase 3 trial. Lancet. 2014;383(9916):533-540.

23. Frieling H, Hillemacher T, Ziegenbein M, Neundörfer B, Bleich S. Treating dopamimetic psychosis in Parkinson's disease: structured review and meta-analysis. Eur Neuropsychopharmacol. 2007;17 (3):165-171. doi:10.1016/j.euroneuro.2006.08.007

24. Nichols MJ, Hartlein JM, Eicken MG, Racette BA, Black KJ. A fixed-dose randomized controlled trial of olanzapine for psychosis in Parkinson disease. F1000Research. 2013;2:150. doi:10.12688/ f1000research.2-150.v1

25. Zhang H, Wang L, Fan Y, et al. Atypical antipsychotics for Parkinson's disease psychosis: a systematic review and meta-analysis. Neuropsychiatr Dis Treat. 2019;15:2137-2149. doi:10.2147/NDT.S201029

26. Meltzer HY, Mills R, Revell S, et al. Pimavanserin, a serotonin(2A) receptor inverse agonist, for the treatment of parkinson's disease psychosis. Neuropsychopharmacology. 2010;35(4):881-892. doi:10.1038/npp.2009.176

27. Castro VM, Clements CC, Murphy SN, et al. QT interval and antidepressant use: a cross sectional study of electronic health records. BMJ. 2013;346:f288. doi:10.1136/bmj.f288

28. Marek GJ, Carpenter LL, McDougle CJ, Price LH. Synergistic action of 5-HT2A antagonists and selective serotonin reuptake inhibitors in neuropsychiatric disorders. Neuropsychopharmacology. 2003;28 (2):402-412. doi:10.1038/sj.npp.1300057

29. Fava M, Evins AE, Dorer DJ, Schoenfeld DA. The problem of the placebo response in clinical trials for psychiatric disorders: culprits, possible remedies, and a novel study design approach. Psychother Psychosom. 2003;72(3):115-127. doi:10.1159/000069738

30. Rybin D, Doros G, Pencina MJ, Fava M. Placebo non-response measure in sequential parallel comparison design studies. Stat Med. 2015;34(15):2281-2293. doi:10.1002/sim.6494

31. Posner K, Brown GK, Stanley B, et al. The Columbia-suicide severity rating scale: initial validity and internal consistency findings from three multisite studies with adolescents and adults. Am J Psychiatry. 2011;168(12):1266-1277. doi:10.1176/appi.ajp.2011.10111704
32. Shelton RC, Fava M, Freeman MP, et al. Effect of adjunctive pimavanserin on suicidal ideation in patients with major depression: analysis of the CLARITY study. $J$ Affect Disord. 2020;277:478-485. doi:10.1016/j.jad.2020.08.051

33. Perlis RH, Laje G, Smoller JW, Fava M, Rush AJ, McMahon FJ. Genetic and clinical predictors of sexual dysfunction in citalopram-treated depressed patients. Neuropsychopharmacology. 2009;34(7):1819-1828. doi:10.1038/npp.2009.4

34. Clayton AH, El Haddad S, Iluonakhamhe JP, Ponce Martinez C, Schuck AE. Sexual dysfunction associated with major depressive disorder and antidepressant treatment. Expert Opin Drug Saf. 2014;13(10):1361-1374.

35. Ishak WW, Christensen S, Sayer G, et al. Sexual satisfaction and quality of life in major depressive disorder before and after treatment with citalopram in the STAR*D study. J Clin Psychiatry. 2013;74 (3):256-261. doi:10.4088/JCP.12m07933

36. Fava M, Rush AJ, Alpert JE, et al. Difference in treatment outcome in outpatients with anxious versus nonanxious depression: a STAR*D report. Am J Psychiatry. 2008;165(3):342-351. doi:10.1176/appi. ajp. 2007.06111868

37. Ionescu DF, Niciu MJ, Henter ID, Zarate CA. Defining anxious depression: a review of the literature. CNS Spectr. 2013;18 (5):252-260. doi:10.1017/S1092852913000114

38. Cutler AJ. The role of insomnia in depression and anxiety: its impact on functioning, treatment, and outcomes. J Clin Psychiatry. 2016;77 (8):e1010. doi:10.4088/JCP.14076tx3c

39. Nutt D, Wilson S, Paterson L. Sleep disorders as core symptoms of depression. Dialogues Clin Neurosci. 2008;10(3):329-336.

40. Fava M. Daytime sleepiness and insomnia as correlates of depression. J Clin Psychiatry. 2004;65(Suppl 16):27-32.

41. Kaida K, Takahashi M, Akerstedt T, et al. Validation of the karolinska sleepiness scale against performance and EEG variables. Clin Neurophysiol. 2006;117(7):1574-1581. doi:10.1016/j.clinph.2006.03.011

42. Jha MK, Fava M, Freeman MP, et al. Effect of adjunctive pimavanserin on sleep/wakefulness in patients with major depressive disorder: secondary analysis from CLARITY. J Clin Psychiatry. 2020;82(1). doi:10.4088/JCP.20m13425.

43. Jha MK, Minhajuddin A, Fatt CC, et al. Association between irritability and suicidal ideation in three clinical trials of adults with major depressive disorder. Neuropsychopharmacology. 2020;45 (13):2147-2154. doi:10.1038/s41386-020-0769-x

44. Jha MK, Minhajuddin A, South C, Rush AJ, Trivedi MH. Irritability and its clinical utility in major depressive disorder: prediction of individual-level acute-phase outcomes using early changes in irritability and depression severity. Am J Psychiatry. 2019;176 (5):358-366. doi:10.1176/appi.ajp.2018.18030355

45. Minhajuddin A, Jha MK, Chin Fatt C, Trivedi MH. Psychometric properties of the concise associated symptom tracking scale and validation of clinical utility in the EMBARC study. Psychiatry Res Clin Pract. 2020;appi.prcp.20190041.

46. Jha MK, Minhajuddin A, Chin Fatt C, Trivedi MH. Improvements in irritability with sertraline versus placebo: findings from the EMBARC study. J Affect Disord. 2020;275:44 47. doi:10.1016/j.jad.2020.06.021

47. News Release; 2020. Available from: https://ir.acadia-pharm.com/ news-releases/news-release-details/acadia-pharmaceuticalsannounces-top-line-results-phase-3-0?field_nir_news_date_value $\%$ $5 \mathrm{Bmin} \% 5 \mathrm{D}=$. Accessed November 02, 2020.

48. Trivedi MH, South C, Jha MK, et al. A novel strategy to identify placebo responders: prediction index of clinical and biological markers in the EMBARC trial. Psychother Psychosom. 2018;87 (5):285-295. doi:10.1159/000491093 


\section{Publish your work in this journal}

Drug Design, Development and Therapy is an international, peerreviewed open-access journal that spans the spectrum of drug design and development through to clinical applications. Clinical outcomes, patient safety, and programs for the development and effective, safe, and sustained use of medicines are a feature of the journal, which has also been accepted for indexing on PubMed Central. The manuscript management system is completely online and includes a very quick and fair peer-review system, which is all easy to use. Visit http://www. dovepress.com/testimonials.php to read real quotes from published authors.

Submit your manuscript here: https://www.dovepress.com/drug-design-development-and-therapy-journal 